\title{
El viaje por el rio Magdalena, del Consejero Lisboa, embajador del imperio del Brasil en 1853
}

Alejandro Mendible Zurita Universidad Central de Venezuela

Recibido: Septiembre de 2014

Aceptado: Febrero de 2015

\section{Resumen}

En el trabajo se destaca el testimonio del Consejero Lisboa, diplomático del Imperio del Brasil y viajero, quien navegó por el río Magdalena en 1853, y realizó las primeras gestiones en el establecimiento de los límites del Brasil con la Nueva Granada y Venezuela, países con estratégicas localizaciones entre la región amazónica brasileña y el mar Caribe. En la actualidad, las relaciones de estos países experimentan un cambio transformador positivo por la valorización de la parte norte del continente.

Palabras claves: Río Magdalena-Consejero Lisboa-Viajero-Nueva GranadaImperio del Brasil

\section{Voyage by the Magdalena river, the Consejero Lisboa, Ambassador the of the Brazil in 1853}

\begin{abstract}
The article highlights the testimony of Counselor Lisboa, a diplomatic and a traveler from the Brazilian Empire who navigated through the Magdalena river in 1853 and also took the first steps to establish the limits between Brazil and the Nueva Granada and Venezuela, the last two countries having strategic positions in the Amazonic Brazil an the Caribbean Sea. Actually, the diplomatic relations between these countries undergoes a very important and positive change due to the revalorization of the north part of the continent.
\end{abstract}

Key words: Rio Magdalena- Consejero Lisboa-traveler-New Granada-Imperio of the Brazil

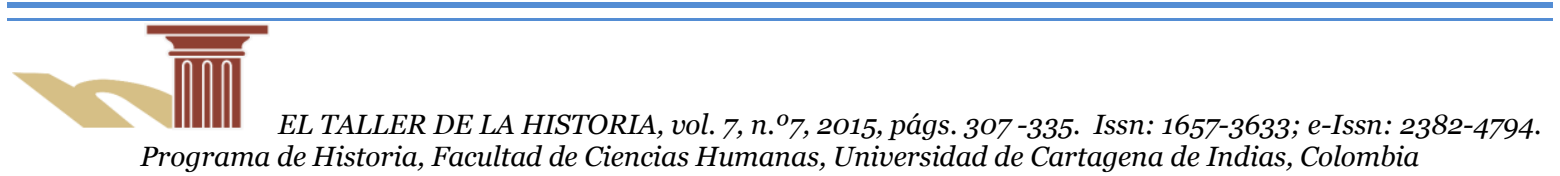


El río Magdalena ha sido el gran articulador natural del proceso histórico colombiano desde los tiempos coloniales, y en el pasado funcionó como facilitador de su economía, de su comercio y de la cultura en todas sus manifestaciones. ${ }^{1}$ De esto dieron cuenta los diferentes viajeros, en su mayoría europeos, que navegaron por sus "marrones y arrugadas corrientes". Entre ellos, destacamos como el propósito del siguiente trabajo el realizado por el Embajador del Imperio del Brasil, el Consejero Lisboa en 1853, como el representante de la cultura luso-americana que disputó con la hispanoamericana el dominio de la América del Sur hasta principios del siglo XIX. Durante la Independencia, su país optó por la transición pacifica y continuó con el sistema monárquico, lo que le permitió conservar su territorio colonial, mientras en la Nueva Granada el proceso fue violento, adoptó el sistema republicano y solamente pudo preservar partes importantes del extenso territorio colonial español, del cual sólo quedaron como colonias las islas de Cuba y Puerto Rico, en el mar de las Antillas.

La obra escrita y la actividad diplomática desplegada por el primer embajador del Imperio brasileño, el Consejero Lisboa, ${ }^{2}$ en los países que antes fueron par-

\footnotetext{
${ }^{1}$ Las referencias son múltiples y variadas. Para muchos, desde el nacimiento del río Magdalena, en el Paramo de las Papas, hasta su desembocadura en el mar Caribe hay música y baile a lo largo de $1525 \mathrm{kms}$. El Centro de Documentación Musical inaugurado, en Barranquilla, en el 2006, tiene una biblioteca especializada en la música sobre el Magdalena. En la referencia a la poesía puede citarse la selección de poemas de Julio Flórez, los importantes trabajos realizados por el Profesor de la Universidad del Atlántico, Ariel Castillo Mier. Además de importantes poetas extranjeros como el cubano Nicolás Guillén y el chileno Pablo Neruda. En la literatura bastaría con mencionar las obras de Gabriel García Márquez, El Amor en los tiempos de cólera, Bogotá, Editorial Oveja Negra, 2000, El General en su laberinto, Bogotá, Editorial Oveja Negra, 2000. En esta última novela con su magistral estilo asocia los viajes del Libertador Simón Bolívar con el río, destacando momentos importantes de su vida, pero, el cuarto, cuando navega por el Magdalena y se dirige a al encuentro con la muerte en la ciudad de Santa Marta es el tema central de la obra. El escritor venezolano Mariano Picón Salas en su novela Pedro Claver, el santo de los esclavos, señala, "el río grande de la Magdalena unía con su enorme cinturón luminoso. Con sus riveras de selva y bejucos floridos, con la algazara animal de sus papagayos, monos y guacharacas, con el silencio calcinado de sus bancos de arena, la inmensa distancia que media entre el mar y los altiplanos”. Mariano Picón Salas, Pedro Claver, el santo de los esclavos, Caracas, Biblioteca Ayacucho, 1992, p.51.

2 El titulo de "Consejero" como se usaba en el lenguaje diplomático de la época se refería al Secretario de mayor categoría de la Embajada o Legación brasileña, aunque, Lisboa, también, se desempeño como "Encargado de Negocios".
} 
te integrante de la republica de la Gran Colombia, ${ }^{3}$ puede ser considerada como la de un precursor que avizoraba la importancia que tendría para su país su frontera norte, así como las relaciones de Venezuela y Colombia los vecinos que ocupan un espacio estratégico que se extiende hasta la cuenca del Caribe. En la década de 1850, cuando Lisboa realizó su trabajo diplomático, las fronteras en el interior al norte del continente sudamericano separaban territorios nacionales muy distantes y dotados de poco dinamismo económico. En la actualidad a más de siglo y medio después, en 2014, se ha producido un cambio histórico de todo orden transformador de los antiguos límites en "fronteras vivas" y de cooperación binacional, que nos permite apreciar la importancia creciente de la influencia del auge económico brasileño, así como el mejoramiento constante de sus relaciones con los dos países bolivarianos antes señalados y de los tres pequeños localizados en el escudo Guayanés, antiguos enclaves coloniales europeos y hoy independientes con la excepción de la Guayana francesa. ${ }^{4}$ El desbordamiento de Brasil y su presencia actual en el caribe en el siglo XXI ya lo presenta como un potencial contrincante de los poderes coloniales europeos y de los Estados Unidos. ${ }^{5}$

Para ampliar de manera breve el anterior señalamiento, visto desde el presente, podemos decir que en la segunda década del siglo XxI, Brasil ha logrado cohesionar y uniformar el proyecto de mercado regional formado en 1991, el Mercosur, impulsado inicialmente por los presidentes democráticos, después de las dictaduras militares, Carlos Menen de Argentina y José Sarney de Brasil e integrado en su fundación por Argentina, Brasil, Paraguay y Uruguay. Lo ha convertido en la actualidad como una expresión de sus objetivos nacionales en Sudamérica y su anterior rival histórico, Argentina es hoy su principal aliado en el Cono Sur - Esta nueva situación le permite al Estado brasileño reorientar sus potencialidades económicas hacia el desarrollo del tradicionalmente desasistido norte. Una muestra de ello se puede ver en el crecimiento de las ciudades de esta parte del país y de los

\footnotetext{
${ }^{3}$ Las repúblicas del Ecuador, Nueva Granada (hoy Colombia) y Venezuela

${ }^{4}$ Guayana británica y Guayana Holandesa o Surinam

${ }^{5}$ Los dos países bolivarianos son Colombia y Venezuela y los localizados en la región de Guayana, donde Brasil comparte con los antiguos enclaves coloniales de Inglaterra, hoy República Cooperativa de Guyana; de Holanda, Hoy Surinam y la Guayana Francesa en la actualidad tiene un estatus de Departamento francés de ultramar. Toda la región de Guayana tiene un área de 2,5 millones de km².
} 
diferentes proyectos de desarrollos que han contribuido para que la región deje de ser expulsora de mano de obra para la región sur y entre en un crecimiento socio económico sostenido. ${ }^{6}$ La tradicionalmente región del Nordeste, considera como la mas deprimida del país, viene creciendo el triple de la media nacional, sólo en los primeros cinco meses de 2014, el crecimiento fue de $4 \%$ y fue responsable de la generación de 2/3 de todos los empleos del Brasil.7 También, desde 1989, el norte del Brasil actúa como el gran promotor del Tratado Amazónico y de los organismos creados, posteriormente, para su ejecución como el Parlamento Amazónico, integrado por representantes de Colombia, Perú, Brasil, Bolivia, Ecuador Surinam, Guayana y Venezuela, quienes se constituyen para valer por la conservación y mejor uso de de los ingentes recursos y potencialidades de la Amazonia, en beneficio de sus pobladores.

Con el ingreso de Venezuela al Mercosur en 2012, éste mercado se convierte en la quinta economía del mundo y Brasil logra conformar su histórica aspiración geopolítica: la de uniformar bajo su control la cara del Atlántico sur. Además, Brasil, aprovecha la plataforma creada por Venezuela para el abastecimiento de petróleo subsidiando a veinte países caribeños mediante Petrocaribe para llegar a esta disputada región y valerse de las estrechas relaciones existentes entre Venezuela y Cuba para ganar el place en la construcción del puerto cubano de Mariel, uno de los mayores del área, localizado a $200 \mathrm{~km}$ de la Florida y desde donde Brasil se le facilita las rutas marítimas en el Caribe. Evidentemente, estos logros lo colocan en una situación privilegiada, después del histórico pronunciamiento de los presidentes Barack Obama y Raúl Castro del 17 de diciembre de 2014 de suspender el bloqueo y reanudar relaciones diplomáticas.

\footnotetext{
${ }^{6}$ Los datos estadísticos sobre el crecimiento de la parte norte del Brasil son variados y abarca desde la construcción de carreteras, represas, electricidad, salud, educación, etc. Para muestra señalamos que la prensa brasileña, (entre ellos, O Globo, Folha de Sao Paulo y Jornal do Brasil) en el mes de diciembre del 2014, a la construcción de un complejo portuario en Mirtituba y Barbacena, constituye una salida por el norte de la actividad comercial del Brasil en una monta en toneladas que tiende a equiparase al puerto de Santos en el Sur. Por tal motivo algunos órganos de prensa lo señalan como, "el corredor norte es la mayor obra de expansión del país".

7 Los datos son suministrados en el portal del Instituto Lula, en Google, en la página "A verdade sobre o Nordeste". Consulta: 10 de diciembre de 2014.
} 
Regresando al siglo XIX encontramos que, durante la época del Imperio (1822-1889) una de las cinco regiones geográficas del Brasil, la del norte fue quedando rezagada del desarrollo del resto del país, el cual se orientaba hacia el sur. En este cambio en la primera región dominaban los espacios vacíos con un lento crecimiento demográfico y una producción económica imperceptible en las arcas nacionales, de la distante capital de Río de Janeiro frente al Atlántico Sur mayormente involucrada con las actividades de la cuenca del río de la Plata.

La parte norte del Brasil, en 1621 durante el período colonial fue dividida entre las provincias de Amazonas y la del Pará pero, sólo, alcanzó interés para la corona durante las reformas emprendidas por el Marques de Pombal (1750-1777), primer ministro del Rey José I y una de las figuras más controvertidas y carismáticas de la Historia Portuguesa. ${ }^{8}$ Antes de la independencia en 1822 , la corona mantuvo la región dependiendo de manera directa de Lisboa y separada del resto del Virreinato del Brasil. En el estallido de la independencia se muestra reticente de reconocer la nueva autoridad del príncipe Pedro I, como Libertador y primer Emperador del Imperio Brasileño, por lo cual fue reducida por la fuerza militar. Posteriormente, después de concretada la independencia durante el inicio del segundo imperio, cuando el Emperador Pedro II todavía no tenía la edad requerida por la constitución, de 1824, para ejercer el reinado, en el periodo de las Regencias se produce una revolución violenta en la provincia de Pará que se extiende hasta región amazónica, la Cabanagem (1835-1840), con tendencia secesioncitas del resto del país.

Durante el imperio brasileño los grandes espacios de la región amazónica compartidos con las repúblicas de Venezuela, Ecuador, la Nueva Granada, Bolivia y Perú conformaban en el centro del continente sudamericano un enorme Hinterland, sin delimitar de manera apropiada entre estos países y con escasa presencia de sus respectivos Estados. Esta cuestión experimenta un giro a partir de 1850, cuando se manifiesta la codicia internacional por controlar la región, en especial por parte del colonialismo Inglés y más tarde por el expansionismo de Estados Unidos. En ese momento, la Cancillería del Imperio del Brasil se encontraba en

\footnotetext{
${ }^{8}$ El favorito del rey, el Marqués de Pombal. Expulso a los jesuitas de la Región Amazónica, nombro a su hermano encargado de las provincia del Pará, cuya capital era el puerto de Belem e incentivo el comercio mediante la creación de compañías para la exportación de la castaña y otros productos,
} 
mejores condiciones para percibir las potenciales amenazas externas que los ministerios de política exterior de sus países vecinos republicanos. Por cuanto, Brasil se encontraba estabilizado y en calma, cuestión que contrastaba con la inestabilidad creada por el fenómeno del caudillismo y la fragilidad institucional presentes en los países bolivarianos, también, por la preservación en la Cancillería brasileña de la actividad profesional de sus funcionarios, quienes pudieron mantener la continuidad administrativa durante el traumático proceso de independencia en 1822 y les fueron respetados sus títulos nobiliarios, cuestión que en las nuevas repúblicas fueron abolidos. El imperio brasileño formó una nobleza de servicio y no de sangre, como en Europa, ya que, utilizo los trabajos prestados al Estado en un canal de ascenso social, de tal forma, que un funcionario eficiente como lo era el Consejero Lisboa, pudo conseguir un título de noble.

Cuando Brasil adquiere su independencia tiene una situación confortable en relación al resto de países del Continente, por cuanto conserva todo su antiguo territorio colonial. Sin embargo, en el pasado sólo tenía que lidiar con la corona española y en el período independiente, con cerca de 16 mil kms. de fronteras, debería hacerlo con diez Estados limítrofes diferentes. Este desafió necesitaba profesionalismo y dedicación, cuestión que Miguel María Lisboa, primer embajador en Venezuela, Ponte Ribero, primer embajador en Perú y muchos otros diplomáticos representaron en sus funciones. Tomando, por referencia la delicada situación amazónica la elite del imperio a mediados de 1850 establece una doctrina de límites que asocia con el respeto al Uti Possidetis, opta por las negociaciones bilaterales y acepta el arbitraje en caso de surgir algún impase. ${ }^{9}$ Aplicando esta metodología el imperio brasileño se acerca a las elites gobernantes de los países vecinos, en momento que se mostraba, en estos, el interés por la implantación de los estados nacionales, de lo cual los límites era un aspecto esencial. Determinado por este contexto se orienta la labor del embajador brasileño Miguel María Lisboa, mejor conocido como Consejero Lisboa.

9 Amado Luiz Cervo y Clodoaldo Bueno, História da política exterior do Brasil, Brasilia, Editora Universidade de Brasilia, 2008, pp.87-107. 
En el presente trabajo. nos interesa como ya señalamos, destacar el viaje de Lisboa, en 1853, por el río Magdalena, el principal de la Nueva Granada y el cual incluye en los capítulos XVIII y IX de su interesante libro titulado: Relación de un viaje a Venezuela Nueva Granada y Ecuador (terminado y publicado en Bruselas, Bélgica, en julio de 1865). Poco tiempo después, el 13 de mayo de 1866, Joaquín M. Machado de Assis, considerado como el más ilustre de los escritores brasileños, escribe una columna en el Diario de Río de Janeiro y comentarios sobre el libro destacando su importancia para los lectores de su país conocer las condiciones de vida de países vecinos de cara al mar Caribe. ${ }^{10}$ En 1954, el sugestivo libro es traducido al español y publicado por primera vez por las ediciones de la Presidencia de la República de Venezuela. En 1983 sale una segunda edición en español (con una nueva traducción), publicada por el Fondo Cultural Cafetalero de Colombia, La más reciente publicación es la elaborada, en 1992, por la Biblioteca Ayacucho de Venezuela, en su colección paralelos, con un prologo explicativo del diplomático brasileño Joao Hermes Pereira de Araujo y una introducción del intelectual y geógrafo Pascual Venegas Filardo.

Para el momento de la llegada de Lisboa a la región del Caribe de la Nueva Granada las provincias de Cartagena y de Mompox formaban el Estado Soberano de Bolívar, con capital en Cartagena e integrado entre otros distritos por el de Barranquilla, y era cuando el gobernador del Estado, "el general Juan José Nieto trazaba con optimismo una visión de la región". ${ }^{11}$ Sin embargo, la realidad de los hechos indicaba lo contrario, por cuanto la región de la costa se encontraba en decadencia por los efectos de la guerra de independencia cuyas consecuencias destructivas sobre la población aún eran visibles, la contienda bélica quebranto la economía de manera severa y le asesto un golpe importante a la sociedad estamental, pero la estructura de la tierra permaneció acentuando la desigualdad social. A las bajas tasas de crecimiento de la población se le sumaba una sobre mortalidad creada por las epidemias: entre ellas las del cólera desatada a partir del mes de julio de

\footnotetext{
10 Machado de Assis (1839-1908), publicó en varias revistas y periódicos de Río de Janeiro, entre ellos; Revista Ilustración Brasileira. Entre sus obras más destacadas se encuentran: Memorias Póstumas de Bras Cubas, Quicas Barbas y Dom Casmurro. También. escribió más de doscientos cuentos, poesía y teatro. Fue el primer presidente de la prestigiosa Academia Brasileira de letras en 1897.

11 Eduardo Posada Carbó, "Progreso y estancamiento 1850-1950", en Adolfo Meisel (ed.), Historia económica y social del caribe colombiano, Bogotá, Universidad del Norte, 1994, p.237.
} 
1850, la fiebre amarilla y la lepra. ${ }^{12}$ Además, la decadencia se patentiza con el desplazamiento del poder político hacia la región andina, dominada por Bogotá.

Lisboa señala que "la universidad del segundo distrito,[la actual fue creada en 1877] tiene asiento en Cartagena, pero, como la de Bogotá, esta en completa decadencia"13 y sobre la ciudad dice que se encuentra localizada, "entre la desembocadura del Magdalena, que queda al este, y el golfo de Darién, en el que desagua el río Atrato, era, en tiempos pasados, una de las plazas de guerra más fuertes y su puerto uno de los más animados de la América española. Consisten sus fortificaciones en una espesa y sólida muralla, construida de piedra de cantería, la cual ciñe el oblongo recinto de la ciudad y está guarnecida con baluartes y ángulos salientes, con un revellín o media luna que defiende, por la parte de afuera, el suburbio de Ximani. Sus obras externas son el fuerte de San Felipe o San Lázaro, situado sobre una colina al este que domina la ciudad". ${ }^{14}$ Esta semblanza de las condiciones de la ciudad son un tanto diferentes a la decadente condiciones formuladas por Theodore Mollien en 1823, cuando observa: "Cartagena presenta el aspecto lúgubre de un claustro; largas galerías, columnas bajas y toscas, calles estrechas y sombrías en razón al saliente de los tejados que surten la mitad de la luz; la mayor parte de las habitaciones están sucias, llenas de humo, tienen aspecto mísero, y cobijan seres que están más sucios, más negros y más miserable aún". ${ }^{15}$

Por otra parte, Lisboa en sus apreciaciones sobre Cartagena Introduce datos históricos no excepto de humor cuando dice que, "al Gobierno español le costó la fortificación de esta plaza 20.000.00o de pesos, que son 40.000 contos de nuestra moneda [brasileña]. Cuéntese que Felipe II, al recibir en Aranjuez la cuenta del dinero en ella gastado, tomó un anteojo de larga vista y llegándose a la ventana em-

\footnotetext{
12 Eduardo Posada Carbó, “Progreso y estancamiento 1850-1950”, p.251.

${ }^{13}$ Consejero Lisboa, Relación de un viaje a Venezuela, Nueva Granada y Ecuador, Caracas, Ediciones de la Presidencia de la República de Venezuela, 1954, p.310.

${ }^{14}$ Consejero Lisboa, Relación de un viaje a Venezuela, Nueva Granada y Ecuador, p.316.

15 Ministerio de Educación Nacional Colombia, Viajeros en la Independencia, Bogotá, Colombia, 2009, p.25
} 
pezó a examinar el horizonte con el; al preguntarle un cortesano que es lo que S. M. mira, respondió el rey: Estoy viendo si diviso las fortificaciones de Cartagena" “Como?-le replicó el valido-¿Desde esta distancia?” ¿Por que no? -replico Felipe- A juzgar por lo que me cuestan, deben ser tan altas que no sería extraño que las divisásemos desde aquí". ${ }^{16}$

La lectura del libro de Lisboa nos sugiere la existencia de por lo menos dos condiciones temporales en el proceso de la escritura del texto. La primera corresponde a la etapa de cuando permanece en Venezuela por diez años y un año adicional en el viaje por Nueva Granada y Ecuador, lo que supone una suerte de diario en el cual, el Consejero recoge sus observaciones de lo que pudo ver y vivir. Luego podemos interpretar una segunda parte, la cual pertenece a un tiempo posterior: donde la memoria y la reflexión del autor actúan en la revisión del texto original. Esta segunda parte se extiende hasta el mes de julio de 1865 cuando Lisboa escribe el prologo final de la obra en la ciudad de Bruselas.

El texto es un valioso testimonio elaborado por un representante de la elite brasileña quien al observar los valores y costumbres hispanoamericanas le permite establecer el contraste. También, contiene información que va desde las descripciones geográficas y naturales, pasando por la vida cotidiana y apreciaciones sobre acontecimientos políticos en Venezuela, Nueva Granada y Ecuador.

En lo referente al río Magdalena el autor señala que, "desagua en el mar de la Antillas entre las ciudades de Santa Marta y Cartagena". Este es el cauce fluvial Colombiano de mayor extensión y comunica la cuenca del caribe con el mundo andino. El Caribe donde se fraguó el inicio de la conquista española en América, después el escenario de confrontación entre los diferentes imperios coloniales europeos y finalmente, donde se fraguo la independencia. La Andina la cede de las más elevadas civilizaciones americanas en América del Sur, como la cultura Muisca y en especial la incaica.

Lisboa era un hombre culto y bien preparado que iba como un enviado de buen oficio a Bogotá y Quito, con el propósito de negociar la fijación de un tratado de límites y de libre navegación por el río Amazonas. Estas gestiones se enmarcan

\footnotetext{
${ }^{16}$ Consejero Lisboa, Relación de un viaje a Venezuela, Nueva Granada y Ecuador, p.317
} 
en la necesidad del establecimiento de las fronteras como una necesidad importante para el establecimiento de los estados nacionales sudamericanos. Esta cuestión histórica que se manifiesta a partir de la década de 1840 es muy diferente a la de la época colonial cuando en el siglo XVI, cuando las fronteras imaginarias entre los corona ibéricas eran transgredidas por los intrépidos bandeirantes lusoportugueses procedentes de la costa Atlántica en Sao Paulo en una agresiva acción expansionista hacia el interior de Sur América, que movían las líneas españolas a favor de Portugal.

En la obra de Lisboa encontramos la curiosidad del autor por el conocimiento de variados eventos y situaciones de la vida cotidiana por lo cual se convierte en un gran testimonio de las condiciones de vida existentes en estos países a mediados del siglo XIX. Toma de referencia lo señalado por otro gran viajero de la misma época, el venezolano Francisco Michelena y Rojas, conocido como "el viajero universal", ${ }^{17}$ quien destaca: "La misión de un viajero, si quiere llenarla debidamente se hace indispensable que, al narrar los hechos y exponer sus apreciaciones, lo haga con sinceridad y los compare de la más severa verdad”. ${ }^{18}$ En referencia a la importancia histórica de la actividad del viajero en la historia de América, que para el historiador colombiano, Jaime Jaramillo Uribe se convierte en "la visión de los otros”. ${ }^{19}$ Esta visión surge del descubrimiento del Nuevo Mundo en el siglo XV,

17 Era miembro de una prominente familia de los valles de Aragua, en Venezuela, involucrada en la guerra de independencia. Su hermano Santos Michelena y Rojas fue el negociador del primer tratado de límites con la Nueva Granada, (el Tratado Santos Michelena-Lino de Pombo), el cual fue rechazado por el Congreso Nacional en 1833. Después fue Ministro de Hacienda y de Relaciones Interiores y fue asesinado en el asalto al Congreso al inicio del gobierno de los hermanos Monagas, en 1848. Francisco, su hermano, por su gran afición por los viajes fue llamado el "viajero universal", ya que le dio dos veces la vuelta al mundo.

${ }^{18}$ Francisco Michelena y Rojas, Exploración Oficial por la primera vez desde el norte de la América del Sur por ríos, entrando por las Bocas del Orinoco, desde los valles de éste mismo y del Meta, Casiquiare, Río Negro ó Guaynía y Amazonas, hasta Nauta en el Alto Marañón o Amazonas, arriba de las bocas del Ucayali bajada del Amazonas hasta el Atlántico Comprendiendo en ese inmenso espacio los Estados de Venezuela, Guayana Inglesa, Nueva Granada, Brasil, Ecuador, Perú y Bolivia, Viaje a Río de Janeiro desde Belem en el Gran Pará, por el Atlántico tocando en las capitales de las principales provincias del Imperio, En los años de 1853 hasta 1859. Bruselas, A. Lacroix, Verboneckhoven y Cía., Impresores y Editores, 1857, p.43.

${ }^{19}$ El término es compartido con otros historiadores por ejemplo, el venezolano Elías Pino Iturrieta. 
cuando los hechos narrados en los relatos de viajes adquieren el carácter de una fuente y un método que trasformaron la visión de la historia al colocar al europeo ante nuevos hechos, nuevas sociedades y nuevas culturas; lo cual condujo a la creación de varias visiones mitológicas tales como la fabulosa leyenda del "dorado", la existencias de tribus de amazona, de pigmeos y/o la teoría del "buen salvaje".

En los siglos posteriores y en especial durante el siglo XIX, en buena medida, los sucesivos viajeros que vinieron produjeron lo que se ha llamado el "segundo" descubrimiento de América por los europeos y en esta nueva onda se destaca el gran explorador alemán, Alejandro de Humboldt quien escribió un ensaya político sobre El Reino de Nueva España y el notable, Viajes a las regiones equinocciales del Nuevo Continente (1799-1804), por lo que es considerado como "el modelo del viajero científico". Humboldt durante sus viajes de estudios paso por la Nueva Granadas, donde elaboró una Carta del curso del río Magdalena, un mapa y navegó por el río hasta el puerto de Mompox. Cuando el viajero partió de Cartagena, tomó el canal por el cual se comunicaba con el río, el cual se había obstruido de tal manera de arena, que sólo podía pasar una canoa pequeña, pero destaca que, "el dique es muy importante para el comercio entre Cartagena y Santa Fé”. 20

Entre los importantes viajeros destacados por la navegación del río, antes de Lisboa, podemos mencionar también, al diplomático inglés John Potte Hamilton, "Comisario de su Majestad Británica ante la República de Colombia", quien en su libro Viajes por el interior de las provincias de Colombia (1827) describe su experiencia al llegar a la ciudad de Santa Marta, el 1 de diciembre de 1823 señalando que, "la vista de la cordillera de los Andes, en la parte posterior de Santa Marta, es grandiosa y sublime, alguna de las montañas tienen gran altura y por lo tanto se hallan en toda época cubiertas de nieve en la cumbre". ${ }^{21} \mathrm{El}$ comerciante francés Gaspar Theodore Mollien quien llega a Cartagena, escribe el libro, Viaje por la República de Colombia (1823) y señala que, "no hay nada más espantoso que un viaje por el Magdalena”.22 El también francés Jean Baptiste Boussingault, quien en 1824

\footnotetext{
${ }^{20}$ Ministerio de Educación Nacional Colombia, Viajeros en la Independencia, p.25.

${ }^{21}$ Ministerio de Educación Nacional Colombia, Viajeros en la Independencia, p.34.

${ }^{22}$ Ministerio de Educación Nacional Colombia, Viajeros en la Independencia, p.50.
} 
escribe sus Memorias; ${ }^{23}$ el sueco Carl August Gosselman, con su Viaje por Colombia 1825 y 1826 quien califica su viaje por el Magdalena, "como la máxima concentración de dificultades y fatiga" 24 y el francés Auguste Le Moyne que escribe Viaje y estancia en la Nueva Granada (1829-1839). ${ }^{25}$

Lisboa es un funcionario del Estado Brasileño y dentro de sus funciones como Embajador se encontraba la de enviar información escrita al Ministerio de Relaciones Exteriores en Río de Janeiro. Por otra parte, vale destacar que el emperador Pedro II es considerado como el gobernante mejor preparado de los diferentes países americanos del siglo XIX, su educación desde niño fue una tarea del Estado Imperial y en su preparación se encontraba el empeño de escribir de manera correcta. Así, el gobernante cultivo la escritura, manteniendo un diario personal y como fue un gran viajero tanto en el interior de su enorme país, como en lo internacional, viajando a los Estados Unidos, Europa y el Cercano Oriente sobre los cuales llegó a escribir algunos ensayos. Esta conducta marco un estilo dentro de los altos funcionarios del imperio y Lisboa no era indiferente a esta influencia. Evidentemente, también, el libro tenía la finalidad de informar a los lectores brasileños de los detalles y de la impresiones del Embajador durante su gestión por países al norte del río Amazonas. Cuando, Lisboa, lo termina y escribe el prologo en julio de 1865 hace una evaluación de las últimas dos décadas y afirma que, "El interés que tienen los brasileños en conocer el estado social de las Repúblicas que limitan con nosotros, en vez de haber disminuido desde 1853 para acá, ha aumentado. Gracias a la apertura del río Amazonas, nuestro comercio con las que limitan por el Norte y el Oeste se van desarrollando.”26

\footnotetext{
${ }^{23}$ Ministerio de Educación Nacional Colombia, Viajeros en la Independencia, p.67.

${ }^{24}$ Ministerio de Educación Nacional Colombia, Viajeros en la Independencia, p.113.

${ }^{25}$ Ministerio de Educación Nacional Colombia, Viajeros en la Independencia, p.127.

${ }^{26}$ Consejero Lisboa, Relación de un viaje a Venezuela, Nueva Granada y Ecuador, p.7
} 


\section{El Consejero Lisboa: su actuación en contexto histórico}

La navegación del Consejero Lisboa por el Río Magdalena forma parte de un periplo mucho mayor que llevó al personaje a emprender viajes por tres repúblicas diferentes y referirlos en un interesante libro de testimonios de sus impresiones. En Venezuela a donde llega en 1842 saca tiempo para visitar buena parte del territorio nacional y describe el puerto de la Guaira, la ciudad de Caracas, las provincias de Barcelona, Cumana, la isla de Margarita, y los fértiles valles de Aragua. En 1852 emprende viaje a la Nueva Granada y al Ecuador para entregar sus credenciales en Bogotá y Quito como enviado del emperador Pedro II antes los respectivos gobiernos. En ese momento, ya Lisboa era considerado como un "verdadero experto diplomático" en relación al conocimiento de los países antes mencionados. También, por exigencias del Ministerio de Relaciones Exteriores (Itamaraty) escribe una Memoria sobre los Límites entre el Imperio del Brasil y la Guayana Frances, sobre lo cual vale señalar que fue tomada por fuerzas expedicionarias brasileñasportuguesas en 1808 como una respuesta en America del Sur a la incursión de Napoleón en Portugal y los portugueses la mantienen hasta 1817, cuando la sagacidad del celebre diplomático francés Talleyrand, en el Congreso de Viena en 1814, logra un nuevo acuerdo con Portugal para regresar a la aceptación del Tratado de 1797.

Los trámites diplomáticos de Lisboa, como enviado brasileño se producen cuando en los países visitados se manifiesta en las elites gubernamentales una voluntad política tendiente al establecimiento de sus respectivos estados nacionales, lo que le daba importancia a la delimitación espacial de sus límites. En este propósito se valoraba la referencia de la Región Amazónica como un importante territorio vacío a ser compartido el Hinterland sudamericano, aceptando en condominio compartidas. Evidentemente ${ }_{2}$ el Brasil era determinante ${ }_{2}$ en estas negociaciones, por cuanto heredaba los grandes territorios conquistados por Portugal a España en el período colonial y legalizado por los tratados de Madrid de 1750 y el de San Ildefonso en 1777.

El Consejero Lisboa nació en Río de Janeiro el 22 de mayo de 1809, un año después de la llegada del rey portugués Joao VI a ésta ciudad que fue convertida en capital del Imperio, un hecho histórico que le cambió el rumbo al Brasil y lo hace 
diferente al resto de países hispanoamericano. ${ }^{27}$ El Consejero murió en Lisboa, Portugal, donde se desempeñaba como Enviado Extraordinario y Ministro Plenipotenciario, cuando completaba 50 años de servicios diplomáticos, el 8 de abril de 1881. Inició su servicio exterior a los dieciocho años de edad, en Londres, en el año 1828 , trabajando como agregado consular. En 1831 lo envían a Chile como Agregado de Negocios, donde permanece hasta 1838 y durante este tiempo mantuvo amistad con Don Andrés Bello, con quien llegó a concebir ideas sobre la iniciativa de crear una Liga Anfictiónica que retomara la propuesta del Libertador Simón Bolívar en 1825 en el Congreso de Panamá, con el propósito de unir a las diferentes naciones sudamericanas incluyendo al Brasil. En 1842 es designado Encargado de Negocios en Venezuela, y cinco años después, en 1847, regresa por poco tiempo a Río de Janeiro para ocupar en el Ministerio el cargo de Secretario de Extranjería. En 1851 es enviado en misión residente a Bolivia y desde allí parte en gestión especial nuevamente a Venezuela, para en esta oportunidad visitar la Nueva Granada y al Ecuador. Después de manera sucesiva es designado en 1855 Ministro Plenipotenciario en Lima, Perú; en 1859 en los Estados Unidos, en 1865 en Bélgica y finalmente en Portugal.

Sus progenitores eran miembros de la nobleza del Imperio y su linaje se remontaba incluso a la corte portuguesa. Su padre llegó a ocupar el cargo de Ministro de Hacienda del Emperador Pedro I y su madre Doña María Eufrasia de Lima Lisboa una destacada dama de la misma corte. El Consejero se graduó en la Universidad de Edimburgo, Escocia, donde obtuvo un título en Artium Magíster (equivalente en nuestros días a una Maestría en Artes) y en vida llega a ser Miembro Correspondiente al prestigioso Instituto Histórico y Geográfico Brasileño y Socio de la Real Academia de Lisboa. El diplomático contrajo matrimonio en Niteroi, una ciudad muy cercana de Río de Janeiro, con su prima, Doña María de Andrade Pinto, hija de una familia muy vinculada con la Casa Real, con quien tuvo cuatro hijas y un hijo. Que siguió sus pasos como diplomático.

\footnotetext{
${ }^{27} \mathrm{La}$ ciudad puerto durante las reformas de la colonia portuguesa sudamericana que emprende el Marqués de Pombal (1699-1782), primer ministro del Rey José (1750-1777) es designada como la nueva capital del Virreinato del Brasil. En 1808 cuando a familia real llegó era un importante puerto negrero y según un comerciante inglés, John Luccok, citado por Laurantino Gomes, la ciudad tenía una población estimada en 80 mil habitantes de los cuales casi la mitad eran negros esclavos.
} 


\section{Viaje del Consejero Lisboa por los tres países bolivarianos}

Brasil y la Gran Colombia destacaban en el contexto sudamericano de la tercera década del siglo XIX con orígenes, culturas y formas de gobierno diferentes: imperial en el primero y republicano en el segundo. A lo largo del efímero momento histórico que le toco compartir entre 1819 y 1830, no cesaron de intentar tender mutuos puentes en pro de unas relaciones fructíferas para ambas partes. Pero sus descomunales geografías, los no menos inmensos problemas y la breve existencia de la Gran Colombia impidieron la conformación y profundización de la amistad y cooperación deseadas.

Poco tiempo después, entre el intervalo, de la Batalla de Boyacá en la Nueva Granada, en 1819, y la de Carabobo, en 1822, que le dio la independencia a Venezuela se producen los primeros encuentros entre enviados del príncipe Pedro I de Brasil y el Libertador Simón Bolívar. ${ }^{28}$ Así, primero, el dignatario brasileño nombra un ministro plenipotenciario, Luis Sousa Días, que se reúne con Bolívar en la ciudad de Bogotá y después, según refiere el historiador Salcedo Bastardo, el Libertador recibe, en Venezuela, al agente Schmidt, "para tratar con su excelencia eficazmente el negocio de la confederación”. ${ }^{29}$ En relación a éste asunto es menester señalar que, el ministro de relaciones exteriores del Brasil, Pinheiro Ferreira en carta de 1822 al agente Schmidt proponía la creación de una Confederación de la Independencia de las Naciones, declarando: "el más valioso apoyo del propósito del General Simón Bolívar, quien en América es inmenso". ${ }^{30}$ Otro hecho importante fue la invitación formulada por la Gran Colombia al Brasil en diciembre de 1824 para que enviase un delegado al Congreso Anfictiónico de Panamá. El imperio envía un delegado plenipotenciario, Teodoro José Bicardi, pero este no pudo llegar y quedo retrasado en el puerto de Salvador Bahía.

\footnotetext{
${ }^{28}$ En ese tiempo el príncipe Pedro se encontraba delegado por su padre el rey Joao VI, como regente encargado del trono de Brasil y al negarse a acatar la orden de las Cortes de regresar a Portugal, opta por declarar la independencia el 7 de septiembre de 1822.

29 José Francisco de Rocha Pombo, Historia do Brasil, São Paulo, Edicoes Melhoramiento, 1956, p.308.
}

${ }^{30}$ Montalbán n. ${ }^{\circ}$ 32, Caracas, Universidad Católica Andrés Bello, Caracas, 2002, p.107 
Bolívar comprendiendo la importancia que revestía el asunto de los límites, designó una comisión integrada por su pariente el coronel Leonardo Palacios y el cartógrafo Gómez. Esta comisión actuó produciendo un informe y material cartográfico referencial. De la lectura de algunas cartas enviadas por Bolívar a Palacios en 1829 , se evidencia que este lo mantenía informado del desarrollo político del imperio. ${ }^{31}$ En 1830 después de la desintegración de la llamada Gran Colombia, cuando se formaba la Republica de la Nueva Granada se produjo un levantamiento militar por el general venezolano Rafael Urdaneta el cual fue solventado con la actuación de los generales José María Obando y José Hilario López. El incidente representaba el enfrentamiento entre los intereses representados en la personalidad del Libertador Simón Bolívar y la de Francisco de Paula Santander, quien sale fortalecido y es nombrado presidente de la Nueva Granda, aún estando en el exilio y asume plenamente en 1833, para un período regular de cuatro años. Según el historiador norteamericano David Bushnell, "La República de la Nueva Granada se afanó por equiparse con una Constitución formal y una serie de instituciones políticas liberales [sin embargo] ... no solamente adolecía de una débil unidad política: estaba dolorosamente marcada por el subdesarrollo social y económico, o más precisamente por la pobreza extrema y estancamiento". 32 En este contexto se produce el primer intento de acercamiento entre los inicios del segundo imperio del Brasil regido por Pedro II y la Republica de la Nueva Granada, cuando los encargados del Ministerio de Relaciones Exteriores, Aureliano de Souza y Oliveira Coutinho (Vizconde de Sepetiba) designan como Primer Ministro del Brasil en Colombia al Sr. Souza Días. 33

Una década después ante una situación completamente diferente en 1842, Miguel María Lisboa llega a Venezuela durante la segunda Presidencia del General Carlos Soublette, de tendencia conservadora, quien estuvo en el cargo hasta el 20

\footnotetext{
31 José Francisco de Rocha Pombo, Historia do Brasil, p.204.

32 David Bushnell, Colombia una nación a pesar de sí misma, Bogotá, Planeta, 2007, p.117

33 Flávio Mendes de Oliveira Castro, História da organizacao do Ministerio das Relacaoes Exteriores, Brasilia, Editora Universidade de Brasilia, 1983, p.65. El personaje vivía en Bogotá desde la década de 1820.
} 
de enero de 1847. Lisboa se mantuvo bastante activo entre otros asuntos formuló la primera proposición para el establecimiento de un Tratado de Límites en 1844, pero en el Consejo de Gobierno no le dieron curso por considerar que el diplomático no había recibido los plenos poderes e instrucciones necesaria para ello y aprovecharon para ratificar que los límites no pueden ser otros que los fijados en los tratados de 1750 y 1777. Lisboa insistió nuevamente en 1852 pero tampoco fue aprobado por haber quedado la petición "congelada" por diez meses. Por cuanto, el funcionario se encontraba viajando fuera del país y además se considero un informe elaborado por la Comisión de Relaciones Exteriores considerando las imprecisiones y vaguedades geográficas de la propuesta. En tal sentido, recomendaba la exploración minuciosa de la cuenca Hidrográfica del Amazonas y del Orinoco antes de asumir cualquier compromiso que comprometiera la República.

En 1853 Lisboa viaja desde Venezuela a la Nueva Granada en misión diplomática y permanece en la capital, Bogotá, desde el 8 de junio hasta el 11 de agosto de ese año, llevando a cabo conferencias con el Secretario de Relaciones Exteriores, Lorenzo María Lleras. Antes, el 27 de agosto de 1845, el Emperador del Brasil había enviado como Encargado de Negocios en Bogotá a don Manuel de Sequeira Lima, pero, como nos refiere Lisboa, éste murió el 18 de junio de 1848, durante un viaje por el Magdalena. Por tal motivo, el Presidente neogranadino, Mosquera (18451849) no pudo entrar en conversaciones con el Brasil sobre el tratado de límites que se proponía. En el año que Lisboa estaba de visita en la Nueva Granada, el país se encontraba impactado en el plano político por la "Revolución del Medio Siglo". Evento que establecía el tránsito de la dominación conservadora a la hegemonía liberal, que se prolonga hasta 1885 . Como primeros actores de ese movimiento figuran el presidente José Hilario López (1849-1852) y después en 1853 el presidente José María Obando. Lisboa, observa la manifestación del fenómeno político colombiano y recoge el hecho en su libro de manera alarmada y califica a los políticos señalados de "imprudentes Frankenstein" por cuanto, predicaron a una plebe sin instrucción y únicamente acostumbrada al dominio del clero las doctrinas comunistas y socialistas más exaltadas". ${ }^{34}$ También, señala la división en dos bandos creadas por el apasionamiento político entre los que se distinguían por el traje co-

\footnotetext{
${ }^{34}$ Consejero Lisboa, Relación de un viaje a Venezuela, Nueva Granada y Ecuador, p.275.
} 
rriente que usaban, y se titularon cachacos (casacas) y ruanas (ponchos). ${ }^{35}$ Mas adelante cuando comenta la Constitución de 1853 aprovecha para colocar un extenso pie de página donde señala que su libro no es una obra histórica o tratado político pero, de manera pormenorizada elabora una interesante actualización de la evolución de la Nueva Granda hasta 1865.

En la administración de Obando, el Consejero Lisboa, intenta entre los meses de junio y julio de 1853 establecer con el Ministro de Relaciones Exteriores, Lleras, un Tratado de Amistad y Límites, Extradición de Reos y Navegación Fluvial, pero no fue aceptado por el Congreso de la Nueva Granada. En principio, éste país se negó a reconocer el acuerdo acordado en 1851 entre Perú y Brasil sobre sus fronteras Amazónicas que tomaba la línea geodésica de Tabatinga y continuaba la frontera tomando como referencia los ríos Japore, Apaporis y Yavari hasta el río Amazonas. Esta situación se mantuvo por varias décadas hasta que finalmente Colombia acepto la línea geodésica divisoria pero, sólo, será hasta 1932 cuando mediante la guerra con el Perú, el Estado Colombiano hace presencia definitivamente en la Amazonia y rescata una porción territorial que tiene por base el puerto de Leticia sobre el río Amazonas. A partir de entonces queda conformada una frontera tri nacional entre la ciudad de Leticia para Colombia, la de Tabatinga para Brasil y algunas islas y asentamientos de la ribera peruana sobre el Amazonas, en especial Santa Rosa y Rondiña.

La última actividad diplomática de Lisboa es en Ecuador, donde llega al puerto de Guayaquil a bordo del vapor inglés "Bolivia" procedente de Panamá. Después de una permanencia de varios días en ésta ciudad, marcha con destino a Quito, que el llama. "la capital más elevada del mundo". ${ }^{36}$ La escalada desde la costa lo realiza en varias jornadas llenas de incidencias que vincula a pasajes espectaculares tales como los picos nevados del Chimborazo, el Cotopaxi y el Aluceno, de cuya cumbre tuvo una de las "vistas más majestuosas que me recuerdo". Anota las

\footnotetext{
${ }^{35}$ Consejero Lisboa, Relación de un viaje a Venezuela, Nueva Granada y Ecuador, p.275.

${ }^{36}$ Consejero Lisboa, Relación de un viaje a Venezuela, Nueva Granada y Ecuador, pp.389-39o. Sin embargo, reconoce que no es la ciudad más alta de Sur América, por cuanto Potosí, Puno y la Paz están más altas.
} 
condiciones del "desgraciado indio" sujeto a todos los rigores de la esclavitud sin compensación y comenta el comportamiento de dos comunidades indígenas completamente diferentes: los nativos de Napo inclinados al comercio, muy activos en sus actividades, y, los jíbaros peligrosos caníbales reductores de cabezas.

En el capitulo dedicado a la ciudad de Quito cuya población la calcula en 60.000 almas, también, coloca un largo pie de páginas que le permite actualizar la evolución de Ecuador hasta 1865. Después, aclara aspectos del Tratado de 1851 firmado entre Brasil y el Perú, por el cual se había aceptado la línea oblicua y arbitraria del Apopó ris-Taba tinga que desfavorecía al Ecuador y que le negaba el acceso a la región Amazonas. Las consecuencias de esta situación fueron la causa de un largo conflicto limítrofe entre Ecuador y Perú hasta 1998, cuando se alcanza la paz, después de tres violentas guerras cortas en 1941, 1981 y 1995. Consecuentemente, Lisboa no pudo lograr establecer un tratado de límites con Ecuador, pero consigue un acuerdo de navegación y posteriormente, el emperador Pedro II, en reconocimiento a su trabajo diplomático realizado lo distingue con el titulo nobiliario de "Barón de Japura", un calificativo epónimo a uno de los ríos afluentes del Río Negro, que a la vez lo es del Río Amazonas. También, en Quito, Lisboa, aprovecha para tomar contacto con diferentes sectores de la sociedad y del gobierno local y en especial, como era su costumbre tener conocimiento sobre la geografía del meridiano del Ecuador, en Quito, así como, de las observaciones de la expedición de M. de La Condamine. Lisboa, puede ser considerado como el más ilustres visitante latinoamericano de la antigua Colombia, al menos por el valor y brillo de sus testimonios.

\section{La travesía por el rio Magdalena}

El Consejero Lisboa embarca en el puerto de la Guaira, en Venezuela, con dirección a Puerto Cabello, donde llega después de cincuenta horas. De allí se dirige a la isla de Curazao para pasar a Riohacha, en la Guajira y finalmente llegar a la ciudad de Santa Marta, que en ese momento experimentaba un período de prosperidad por cuanto su puerto tenía una amplia dinámica internacional y era el sitio orillero más próximo del río destinado al intercambio y almacenaje de mercancías y el tra- 
fico humano. Será desde este puerto que se dirige en un pequeño barco el "Calamar" para iniciar la subida del río Magdalena hasta la ciudad de Mompox.

Describe como el río desemboca en el mar, cerca de Santa Marta y da cuenta de varios ríos de agua cristalina, como el Manzanares, que es el más próximo a la ciudad y señala otros que vienen de [la] Sierra Nevada, montañas situada al sudeste de la población, que se eleva a una altura de quince mil pies sobre el nivel del mar y cuyas cumbres están cubiertas de nieves perpetuas. Después dice que la ciudad de Santa Marta, "tiene de ocho a nueve mil habitantes, población de razas mixtas, en la que predomina la africana. La fundó en 1525 don Rodrigo de Bastidas [fue la primera ciudad colonial fundada en el Continente suramericano]; sus calles son estrechas, aunque rectas; su clima es ardiente e insano, excepto cuando reinan las brisas frescas que empiezan en diciembre; el puerto estaba antes defendido por tres fuertes, llamados Morro, Betin y San Fernando, hoy desarmados, y la bahía que le sirve de ancladero esta abrigada de los vientos; es tranquila y muy abundante en pesca". 37

Lisboa, destaca la actuación del general francés y masón Pierre Labatut, en 1813, con grado superior al de Simón Bolívar y cuyas valientes actuaciones en la guerra de independencia le merecieron el nombramiento de "comandante general del bajo Magdalena”. Labatut posteriormente se traslado al Brasil y se puso al servio del príncipe Pedro I, quien durante la declaración de independencia en 1822 lo designó comandante de las fuerzas brasileñas que derrotaron la insubordinación de las fuerzas portuguesas en la región de Bahía, en esta empresa alistó esclavos para reforzar sus tropas. ${ }^{38} \mathrm{El}$ otro asunto que Lisboa comenta es el relativo a la muerte del Libertador Simón Bolívar, en la quinta de San Pedro tras llegar "disgustado con la oposición que se le hacia en Bogotá”.

Lisboa visita la ciudad de Cartagena en su viaje de regreso de Bogotá, llegando por el trayecto de Popayán, Pasto y pasando por el brazo artificial del río Magda-

\footnotetext{
${ }^{37}$ Consejero Lisboa, Relación de un viaje a Venezuela, Nueva Granada y Ecuador, p.212

${ }^{38}$ Laurentino Gomes, Como um homen sábio, uma princesa triste e un escoces louco por Pinheiro ajudaram D. Pedro a criar o Brasil - um país que tinha tudo para dar errado, Río de Janeiro, Nova Fronteira, 2010 [1822], pp.202-203.
} 
lena, "el Canal del Dique", construido en 1640. ${ }^{39}$ Comenta la fundación de la ciudad en 1533 por Pedro de Heredia y como durante el reinado de Felipe II (15561598) se transforma en una ciudad fuerte completamente amurallada con la finalidad de proteger de la piratería en las posesiones españolas en el Caribe.

Lisboa realiza su primera jornada de viaje por el río Magdalena por tierra, a caballo hasta la localidad de Puerto Viejo, un lugar insipiente en la Laguna de los Patos que se comunica por su interior con la desembocadura del río para crear la Cienaga. En este trayecto reconoce que las molestias que sufre "no se pueden describir", mencionando el acoso de los mosquitos, los caimanes, el sofocante calor y los frecuentes "tapones" formados por troncos y otros desechos que impiden la navegación de los botes por los ramales del río.

En la población de Remolino logra embarcar en el vapor Manzanares con dirección al puerto de Calamar donde llega después de pasar por la aldea de Salamina y navegar por más de cinco horas. En ésta localidad en la ribera izquierda del río, reseña la existencia de un camino de tierra hasta la ciudad de Cartagena y comenta el intento de un ingeniero americano de construir un canal artificial de Barrancas, en el mismo lugar que los españoles intentaron hacerlo. Más adelante continúa su viaje rumbo a los puertos de Heredia y Sambrano pasando por el de Juncal y en lugar señala un depósito de leña donde la compañía de vapores de Santa Marta abastece sus barcos de paleta. Según el historiador colombiano Adolfo Meisel Roca, en la segunda mitad del siglo XIX "casi un centenar de empresas intentaron consolidarse en el intrincado negocio de la navegación por el río Magdalena" ${ }^{40}$ Por su parte. Lisboa, escribe, a modo de resumir su experiencia general que,

\footnotetext{
${ }^{39}$ Las leyendas románticas dicen que don Pedro Zapata de Mendoza Gobernador de Cartagena, excavo el canal en el año señalado con 2000 trabajadores de pueblos y haciendas vecinas. Pero Zapata de Mendoza excavo muy poco y se unió una ciénaga con otra a hachazos, donde el río Magdalena en lo que hoy es Calameres, hasta la Boca de Barbacoas. (tomado de, "El Canal del Dique es un brazo artificial del río Magdalena”, en El Universal, Cartagena, 19 de diciembre de 2014)

40 Adolfo Meisel Roca, Historia económica y social del caribe colombiano, Bogotá, Universidad del Norte, 1994, p.244. El autor señala la Compañía Colombiana de Transporte, L. Gieseken, la The Colombian Railway and and Navigation y la Naviera Colombiana.
} 
su propósito no era contar una por una las miserables aldeas que guarnecen las orillas del Magdalena, sino dar una idea general de todo el trayecto. ${ }^{41}$

A dieciocho legua y media de Santa Marta señala se encontraba Barraquilla que para 1853 tenía la misma población que la anterior y el dato nos permite especular sobre la situación actual cuando la ciudad localizada dentro del curso del río se ha convertido en la más populosa del caribe colombiano. ${ }^{42}$ Lisboa continua du viaje hasta Vuelta de la Madre de Dios y la ciudad de Honda, donde dice se podía subir hasta la capital de Nueva Granada, Bogotá.

El 15 de mayo de 1853 llega a la ciudad de Mompox, con la cual queda agradado y la describe como, "las torres de numerosas iglesia y las casas encaladas y cubiertas de tejas [...] es una ciudad de ocho mil habitantes, situada en la ribera izquierda del Magdalena; esta dividida en cuadras, tiene algunas casas de dos pisos, las calles son estrechas, sin empedrar, pero con una pequeña acera de ladrillos elevado en palmo sobre el suelo. La orilla del río está guarnecida con paredón de ladrillo que sirve de muelle con su parapeto y con sus escaleras de espacio; todo en estado de decadencia notable. Tiene cuatro iglesias terminadas y una bastante espaciosa en construcción". ${ }^{43}$ La ciudad, "había sido durante la colonia el puente de comercio entre la costa caribe y el interior del país y esto había sido el origen de su opulencia". ${ }^{44}$ Durante su permanencia en la ciudad, Lisboa, comenta una conversación con mineros del Chocó que nos permite pensar el desconocimiento que Lisboa tenía como brasileño de la fauna tropical, ya que preguntaba sobre la existencia de "manadas de elefantes", cuestión que formula en otras oportunidades en el libro.

\footnotetext{
${ }^{41}$ Consejero Lisboa, Relación de un viaje a Venezuela, Nueva Granada y Ecuador, p.223

42 La ciudad tenía en 2005 una población de 1.109.00o habitantes y fue erigida en villa el 7 de abril de 1813. Actualmente es el Distrito industrial y portuario de Barranquilla, capital del Departamento del Atlántico. Esta ubicada sobre la margen occidental del río Magdalena a 7,5 kms. de su desembocadura con el mar Caribe.

${ }^{43}$ Consejero Lisboa, Relación de un viaje a Venezuela, Nueva Granada y Ecuador, p.225.

44 Gabriel García Márquez, El general en su laberinto, p.62
} 
A nueve horas de navegación de Mompox encuentra la ciudad de El Banco y de esta localidad se pasa al Puerto Nacional de Ocaña que es una ciudad importante localizada sobre la cordillera oriental y en donde se celebró en 1828 el celebre Congreso Constituyente con el propósito de reformar la Constitución de Cúcuta y resolver los problemas en la república. Durante el evento se enfrentaron las dos tendencias encontradas en relación a la orientación del país: los centralistas y federalistas apadrinadas la primera por Bolívar y la segunda por Santander. También, señala la existencia de un canal de desagüe del río Lebrija donde tiene su asiento la ciudad de Jirón con la existencia de minas de oro y producción de tabaco. Vale decir que, según el historiador, profesor de la Universidad de Cartagena, Wilson Blanco Romero, el tabaco constituyó, "la primera gran expansión productora de exportación [...] que en términos de sostenibilidad permitió la regularización de la navegación a vapor por el río Magdalena". ${ }^{45}$ Lisboa continúa señalando como más adelante en el trayecto de subida cruzan los afluentes el Upón y el Cárare, por el cual se puede ir a las ciudades de Vélez, Tunja y Bogotá; donde se encuentran importantes minas de carbón.

En las próximas jornadas, Lisboa, pasa por el desagüe del río Regala, cuyo transito es peligroso por la rapidez y cruces de las corrientes, siguen a Angostura donde el vapor tiene que aumentar la fuerza en sus ruedas de propulsión para sortear las dificultades que ofrece el río y llegan al puerto de Nare, "el emporio de las provincias de Medellín y Antioquia". ${ }^{46}$ Señala que en el trayecto entre la ciudad de Remolinos y la de Nare demoró nueve días y de este lugar hacia arriba en el viaje no hay nada que merezca atención especial. Refiere que para llegar a la ciudad de Conejo pasó por el desagüe de los ríos la Miel y el Río Negro, donde cambio de barco con destino a la Vuelta de Madre de Dios, el Bolívar un "Champán", de fondo plano, de doce a quince brazas de largo y tripulado por los llamados "bogas", quienes no pueden trabajar sino desnudos y acompañando sus enérgicos movimientos con un alarido continuado de obscenas palabras. Dice que estos tenían un compor-

\footnotetext{
45 Wilson Blanco Romero, "Tabaco, economía y capitalismo en los Montes de María, 1850-1830", en, El Taller de la Historia n. ${ }^{\circ}$, Universidad de Cartagena, Universidad de Cartagena de Indias, 2009, p.193; Wilson Blanco Romero, Historia de El Carmen de Bolívar y su tabaco en los Montes de María. Siglos XVIII-XX, Editorial Universidad de Cartagena, Cartagena de Indias, 2010.

46 Wilson Blanco Romero, Historia de El Carmen de Bolívar y su tabaco en los Montes de María. Siglos XVIII-XX, p.232.
} 
tamiento irreverente y displicente que les hizo perder varios días de retraso hasta llegar finalmente al Pretalito, último desembarco del río. En este punto, vale señalar que sobre los bogas se puede encontrar referencias en la literatura, la poesía y en los mismos viajeros, Así, por ejemplo, Humboldt ${ }^{47}$ se refiere a ellos expresando, que "es muy pintoresco cuando estas figuras bronceadas de fuerza atlética, avanza poderosamente apoyado en la palanca".

Antes de terminar su travesía fluvial, Lisboa formula un juicio de apreciación a modo de conclusión, señalando que, "el río Magdalena es un magnifico río; en sus fértiles marines se produce con abundancia [...] los bosques que los cubren abundan en preciosas maderas de construcción, resina y tinta, como el cedro, la bisnaga (sic) y el palo-mora". ${ }^{48}$ Seguidamente, señala que desde el último puerto fluvial emplea cuatro días en recorrer cuatro leguas "en una vía que da acceso a una de las más cultas capitales de América del Sur”, señalando su llegada a Bogotá, una ciudad para entonces de 53.903 habitantes. ${ }^{49}$

El río Magdalena constituyó la principal arteria por donde se movía la economía colombiana, hasta que nuevas formas alternas de trasporte por carretera, ferroviario y aéreo durante el siglo xx fueron desplazando la utilización del río. También, la concretización de esta realidad fue reflejando la subestimación del Estado colombiano por la región del Caribe, cuestión que empieza a cambiar en las últimas décadas con el surgimiento de planes para revivir la navegación del río Magdalena. En 1994 el Gobierno de Colombia crea la Corporación Autónoma Regional del Río Magdalena (Cormagdalena) con el objeto de recuperar la navegación y la actividad portuaria de río. En el 2010 el Consejo Nacional de Política Económica y Social -Conpes- elabora un plan propuesto por el Gobierno Nacional para restablecer la navegabilidad del río Magdalena, el cual, se enmarca dentro de las políticas de transporte contenidas en el Plan Nacional de Desarrollo PND- 2010-2014

\footnotetext{
${ }^{47}$ Ministerio de Educación Nacional Colombia, Viajeros en la Independencia, p.34

${ }^{48}$ Consejero Lisboa, Relación de un viaje a Venezuela, Nueva Granada y Ecuador, p.239

${ }^{49}$ Consejero Lisboa, Relación de un viaje a Venezuela, Nueva Granada y Ecuador, p.273. El dato Lisboa lo apoya con el censo de 1848, pero según sus observaciones la ciudad excedía de los 60.000 habitantes
} 
"Prosperidad para Todos".50 En la actualidad la prensa colombiana informa de la firma de un contrato mil millonario entre la firma brasileña Odebrecht y Valarcon de Colombia para iniciar la trasformación del río en una autopista acuática, en lo que se considera el proyecto más ambicioso de la costa, promovido por los esfuerzos de capitales públicos y privados. De manera simbólica, la presencia de capital brasileño para la transformación del río Magdalena se produce a 161 años de la navegación del consejero Lisboa por su curso en procura de establecer las buenas relaciones con su país.

Colombia y Brasil son dos países sudamericanos con desarrollos socio- históricos diferentes lo que constituye una muestra de cómo nuestro continente es diverso. Sin embargo, en el siglo xxi Sur América por la voluntad política de las elites de sus doce diferentes gobiernos finalmente han convenido en crea una región cohesionada, donde los intereses comunes priven sobre los parcializados de los estados- nacionales. La creación de la Unión de Naciones Sudamericana (Unasur), cuyo primer paso se dio cuando todos los presidentes sudamericanos asistieron a la ciudad de Brasilia, en el 2000, para atender el convite del presidente Fernando Henrique Cardozo de los 500 años del descubrimiento del Brasil y en el encuentro se acordó convertir el continente sudamericano en un área de libre comercio. Posteriormente, los nuevos gobiernos continuaron la iniciativa, en una serie de reuniones de cúpulas presidenciales que se iniciaron en la ciudad del Cusco el 2004, en el 2009 en la Isla de Margarita, en Venezuela, se acordó el nombre del ente coordinador de los diferentes estados nacionales sudamericanos y finalmente, en 2011 fue aprobado por unanimidad. ${ }^{51}$ En dicho organismo convergen Colombia y Brasil quienes comparten el sueño de la "patria grande" de Bolívar y aunque existen desigualdades entre los dos países y pertenecen a diferentes mecanismos regionales de integración: Brasil al Mercosur en la cara del Atlántico Sur y Colombia a la Alianza del Pacifico esto no es óbice para ejercer el libre comercio. ${ }^{52}$ Conside-

\footnotetext{
50 htps//www.drip.gov.co/conpes/paginas/compes.aspx

${ }^{51}$ En la reuniones se destacó la participación de los presidentes Néstor Kirchner de Argentina, Ignacio Lula da Silva de Brasil y Hugo Chávez Frías de Venezuela

52 Vide informes de Aladi en: http//www.aladi.org/nsf. aladi/ estudios. nsf/. También, la declaraciones del ministro brasileño de Desarrollo Nacional de Comercio Exterior en www. americaeconomía. com. En el portal de Procolombia se encuentran datos al respecto y en cámara de comercio colombo-brasilera. com/ entre los diferentes datos que suministra da cuenta de una conferencia del
} 
rando todo lo anterior señalamos que el viaje del Emperador del Imperio del Brasil, el Consejero Lisboa, por el río Magdalena, en 1853, para establecer las relaciones diplomáticas entre Brasil y Colombia tiene trascendencia histórica para comprender el nuevo estado de las relaciones sudamericanas.

\section{Bibliografía:}

\section{Fuentes Primarias:}

Boyssingault, Jean Baptiste, Memorias de jean Baptiste Boussingault, Bogotá, Banco de la República, 1994

El Heraldo, Barranquilla, 2014

Gomes, Laurantino, Como uma raina louca, um principe medroso e uma corte corrupta enganaram Napoleon e mudaram a História de Portugal e do Brasil, Sao Paulo, Editora Planeta do Brasil, 2007 [1808].

Gomes, Laurentino, Como um homen sábio, uma princesa triste e un escoces louco por Pinheiro ajudaram D. Pedro a criar o Brasil - um país que tinha tudo para dar errado, Río de Janeiro, Nova Fronteira, 2010 [1822].

Gosselman, Carl August, Viaje por Colombia 1825 y 1826, Bogotá, Banco de la República, 1981.

Le Moyne, Auguste, Viaje y estancia en la Nueva Granada, Bogotá, Incunable, 1985 .

ex presidente Lula en Bogotá, el 4 de junio de 2013, en la cual señala que, hay 44 empresas brasileñas en Colombia cuando en 2006 era sólo 2, y que habían invertido 3.700 millones de dólares en el país. La revista Dinero.com señala que Colombia tenía un comercio de exportación hacia Brasil para marzo de 2014 de 1.500 millones de dólares con un crecimiento de 23,2\% 
Lisboa, Consejero, Relación de un viaje a Venezuela, Nueva Granada y Ecuador, Caracas, Ediciones de la Presidencia de la República de Venezuela, 1954.

Michelena y Rojas, Francisco, Exploración Oficial por la primera vez desde el norte de la América del Sur por ríos, entrando por las Bocas del Orinoco, desde los valles de éste mismo y del Meta, Casiquiare, Río Negro ó Guaynía y Amazonas, hasta Nauta en el Alto Marañón o Amazonas, arriba de las bocas del Ucayali bajada del Amazonas hasta el Atlántico Comprendiendo en ese inmenso espacio los Estados de Venezuela, Guayana Inglesa, Nueva Granada, Brasil, Ecuador, Perú y Bolivia, Viaje a Río de Janeiro desde Belem en el Gran Pará, por el Atlántico tocando en las capitales de las principales provincias del Imperio, En los años de 1853 hasta 1859. Bruselas, A. Lacroix, Verboneckhoven y Cía., Impresores y Editores, 1857.

Ministerio de Educación Nacional Colombia, Viajeros en la Independencia, Bogotá, Colombia, 2009.

Mollien, Gaspard Theodore, Viaje por la República de Colombia, Bogotá, Banco de la República, 1992.

Potter Hamilton, John, Viajes por el interior de las provincias de Colombia, Bogotá, Biblioteca v Centenario Colombiano, 1993.

\section{Fuentes secundarias:}

Blanco Romero, Wilson, “Tabaco, economía campesina y capitalismo en los Montes de María, 1850-1930", en El Taller de la Historia vol.1, n. ${ }^{\circ}$ 1, Cartagena, Universidad de Cartagena, 2009.

Blanco Romero, Wilson, Historia de El Carmen de Bolívar y su tabaco en los Montes de María. Siglos XVIII-XX, Editorial Universidad de Cartagena, Cartagena de Indias, 2010.

Bushnell, David, Colombia una nación a pesar de sí misma, Bogotá, Planeta, 2007

Castro, Flávio Mendes de Oliveira, História da organizacao do Ministerio das Relacaoes Exteriores, Brasilia, Editora Universidade de Brasilia, 1983. 
Cervo, Amado Luiz y Bueno, Clodoaldo, História da política exterior do Brasil, Brasilia, Editora Universidade de Brasilia, 2008.

García Márquez, Gabriel, El Amor en los tiempos de cólera, Bogotá, Editorial Oveja Negra, 2000.

García Márquez, Gabriel, El General en su laberinto, Bogotá, Editorial Oveja Negra, 2000.

Gonzalo Sánchez Molero, José Luis, Felipe II la mirada de un rey 1527-1598, Santa Cruz de Tenerife, la isla libros, 2005.

Huber, Otto, Foster, Mathew y et al, Prioridades de conservación para el Escudo de Guayana, Surinam, Conservation International, 2000.

Mendible, Alejandro, El Consejero Lisboa primer embajador del Imperio del Brasil en Venezuela y su libro de viaje, Caracas, Alcaldía de Caracas y Embajada del Brasil, 2003.

Mejía Tirado, Alvaro, "El Estado y la política en el siglo XIX”, en Nueva Historia de Colombia, Bogotá, Planeta, 1989.

Picón Salas, Mariano, Pedro Claver, el santo de los esclavos, Caracas, Biblioteca Ayacucho, 1992.

Porreno, El Licenciado Baltazar, Dicho y hechos del señor rey Don Felipe II, Valladolid, Imprenta de D. Juan de la Cucata, 1866.

Posada Carbó, Eduardo, "Progreso y estancamiento 1850-1950" en Adolfo Meisel (ed.), Historia económica y social del caribe colombiano, Bogotá, Universidad del Norte, 1994.

Ricardo, Cassiano, La marcha hacia el oeste, México, Fondo de Cultura Económica, 1956.

Rocha Pombo, José Francisco de, Historia do Brasil, São Paulo, Edicoes Melhoramiento, 1956. 
Salcedo Bastardo, José Luis, Andrés Bello Americano y otras luces sobre la independencia, Caracas, Academia de la Historia, 1973.

Soudis Najera, Adelaida, "Ruptura del estado colonial y tránsito hacia la república", en Adolfo Meisel (ed.), Historia económica y social del caribe colombiano, Bogotá, Universidad del Norte, 1995.

Wong Hiu, Alberto, "Sociedad y política en Barraquilla durante el período federal (1857-1886)”, en José Villalón (ed.), Historia de Barranquilla, Barranquilla, Ediciones Uninorte, 2000. 